\title{
Pan-hipopituitarismo de surgimento na infância e diagnóstico diferencial de poliúria
}

\author{
Vivianne Almeida da Nóbrega ${ }^{1}(\mathbb{D})$, Vinícius Almeida da Nóbrega ${ }^{\mathbb{B}}$, Rita de Cássia Viegas Lins Soares ${ }^{1}$
}

\begin{abstract}
RESUMO
O hipopituitarismo é a deficiência de dois ou mais hormônios hipofisários, que se expressa por sintomas dependentes do tipo e grau de déficit hormonal. A adequada condução destes pacientes é de fundamental importância para que não acarrete atraso no crescimento e desenvolvimento, óbito ou mudanças na qualidade de vida dos indivíduos. Objetiva-se, neste estudo, relatar caso clínico de abordagem de paciente pediátrico com pan-hipopituitarismo e descrever o manejo adotado, bem como a importância do acompanhamento pelo endocrinologista pediátrico. Tratase de paciente do sexo masculino, 14 anos, com pan-hipopituitarismo iniciado na infância, secundário ao surgimento de germinoma e ao tratamento realizado para o mesmo. A primeira deficiência hormonal apresentada foi diabetes insipidus, seguida, após a realização de quimioterapia e radioterapia, de múltiplas deficiências. O menor segue em acompanhamento especializado, e faz uso de levotiroxina, desmopressina, somatropina, testosterona e prednisolona. A partir do presente relato, percebe-se a importância do diagnóstico oportuno e da adequada abordagem da criança com pan-hipopituitarismo e de seu seguimento a fim de se manter uma qualidade de vida satisfatória.
\end{abstract}

Palavras-chave: Hipopituitarismo, Cuidado transicional, Germinoma, Conduta do tratamento medicamentoso, Qualidade de vida.

1. Centro Universitário de João Pessoa (UNIPÊ), João Pessoa, (PB), Brasil

2. Universidade Federal de Campina Grande (UFCG), Campina Grande,(PB), Brasil 


\section{INTRODUÇÃO}

O hipopituitarismo corresponde ao déficit de dois ou mais hormônios hipofisários ${ }^{1}$ e persiste por toda a vida do indivíduo ${ }^{2}$. De forma geral, a grande variedade de etiologias do hipopituitarismo representa um desafio ${ }^{3}$. As causas podem ser agrupadas em adquiridas e congênitas, sendo estas últimas associadas a alterações genéticas em 5 a $10 \%$ dos casos $^{4}$. Na população pediátrica, embora estudos que quantifiquem a sua prevalência e principais causas sejam escassos, mostrou-se maior porcentagem da doença decorrente de etiologias adquiridas, especialmente, tumores do sistema nervoso central ${ }^{5,6}$.

O quadro clínico varia conforme a gravidade dos déficits hormonais ${ }^{6}$ e, na presença de manifestações sugestivas, requer-se investigação aprofundada prontamente e diagnóstico oportuno ${ }^{2}$. Ademais, pode haver associação a atraso no crescimento e/ou desenvolvimento, comorbidades cardiometabólicas, respiratórias e osteomusculares, além da perspectiva de má influência na qualidade de vida e óbito, se não houver condução clínica apropriada ${ }^{6}$.

$\mathrm{Na}$ adolescência, o paciente torna-se mais independente dos cuidados parentais, o que leva a necessidade de abordagem médica visando à manutenção do tratamento. Portanto, percebe-se que desordens da hipófise durante tal período, demandam atenção especial e representam um desafio no manejo para que haja um desenvolvimento pleno do indivíduo.

Ao exposto, torna-se interessante a descrição de caso de manejo de paciente com panhipopituitarismo de surgimento na infância, após atraso diagnóstico, reforçando-se a necessidade de investigação apropriada, a partir de manifestações clínicas sugestivas e mantendo-se um cuidado contínuo pelo endocrinologista pediátrico.

\section{RELATO DE CASO}

Paciente do sexo masculino, 14 anos, compareceu à consulta com endocrinologista pediátrico, acompanhado da genitora, para seguimento de panhipopituitarismo, em agosto de 2019.

Relatou-se que, em dezembro de 2014, após mudança de Estado, a criança começou a apresentar poliúria e noctúria, tendo procurado médico, que solicitou alguns exames laboratoriais, tais como hemograma e glicemia em jejum, que não demonstraram alterações. Além disso, segundo a história colhida, o profissional não prosseguiu a investigação do quadro e encaminhou o menor para psicólogo, suspeitando-se que os sintomas eram psicogênicos. Não obtendo bons resultados, buscaram consulta, em maio de 2015, com pediatra, que levantou a hipótese de diabetes insipidus (DI) e direcionou o paciente para endocrinologista pediátrico.

Durante avaliação desse médico, foram solicitados exames, que confirmaram o DI. Nesse contexto, inicialmente, foi estabelecida a poliúria, definida como $40-50 \mathrm{ml} / \mathrm{kg}$ em 24 horas, além de terem sido excluídos hipocortisolismo e hipotireoidismo prévios. Posteriormente, a associação de hipernatremia, osmolalidade sérica maior que 300 $\mathrm{mOsm} / \mathrm{kg}$ e osmolalidade urinária menor que 300 $\mathrm{mOsm} / \mathrm{kg}$, em um contexto de valores de glicemia, escórias nitrogenadas, potássio e cálcio séricos normais, determinou o diagnóstico de $\mathrm{DI}^{8}$.

Nesse cenário, a partir da administração subcutânea de análogo sintético da vasopressina (1-desamino-8-D-arginina vasopressina [DDAVP]), na dose de 1 unidade $/ \mathrm{m}^{2}$, com consequente concentração urinária, permitiu-se a diferenciação da causa do DI como sendo central.

O paciente foi medicado com desmopressina nasal, e solicitou-se ressonância nuclear magnética (RNM) do crânio, realizada sem contraste e que apontou presença de hamartoma de cerca de 1 centímetro, próximo à hipófise. $O$ paciente foi, então, encaminhado para neurocirurgião na mesma cidade. Dada a insegurança no diagnóstico, marcaram consulta com outro neurocirurgião. Realizada nova RNM de crânio, dessa vez com contraste, que evidenciou germinoma.

Em julho de 2015, após consulta com o neurocirurgião, a criança foi encaminhada para oncopediatra. Solicitou-se punção liquórica para pesquisa dos marcadores tumorais, beta-HCG, AFP (alfa-fetoproteína) e nova RNM. Os marcadores vieram negativos e a RNM reforçou a hipótese de germinoma suprasselar (Figura 1).

Em seguimento, requisitou-se biópsia de região suprasselar, por neuroendoscopia, e o diagnóstico de germinoma foi confirmado. A quimioterapia foi iniciada, em setembro de 2015, com etoposida e carboplatina, sendo finalizada em novembro. Posteriormente, realizou-se radioterapia em arco modulada volumetricamente (VMAT), sendo a primeira fase de 10 frações x 180cGy em sistema ventricular (com DT $=18 \mathrm{~Gy}$ ) e a segunda (boost) com 7 frações em lesão com margens ( $D T=30.6 \mathrm{~Gy}$ ).

Após seis meses do término do tratamento, 0 menor apresentou cansaço, inapetência e dores nas pernas. Passou por endocrinologista, que constatou 

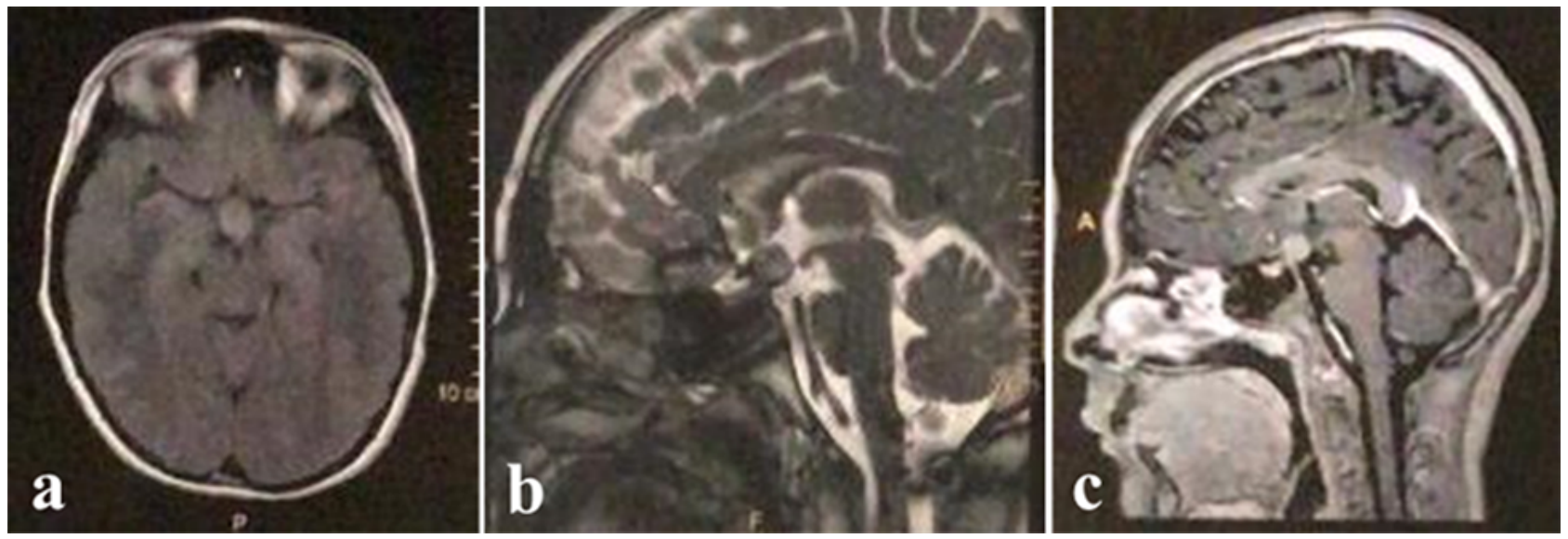

Figura 1. RNM de crânio, julho de 2015. Lesão expansiva, arredondada em região suprasselar, medindo 1,0 x 1,3 x 0,7 cm, apresentando isossinal em T1 (a), leve hipersinal em T2 (b) e grande impregnação pelo método de contraste magnético (c). Não se observam planos de clivagem entre a lesão e a haste hipofisária. (a) Corte axial; (b) e (c) Corte sagital. Fonte: Autores.

hipocortisolismo por déficit de ACTH (hormônio adrenocorticotrófico), prescrevendo prednisolona. Subsequentemente, as deficiências hormonais diagnosticadas foram o hipotireoidismo central, déficit de hormônio do crescimento e hipogonadismo (Figura 2).

Atualmente, o paciente usa levotiroxina $75 \mathrm{mcg} /$ dia, desmopressina spray nasal $0,1 \mathrm{mg} / \mathrm{mL}$ ( 1 jato em cada narina, cerca de 2 vezes ao dia), somatropina 7,2 UI/ dia, testosterona $100 \mathrm{mg} / \mathrm{mês}$ e prednisolona $9 \mathrm{mg}$, pela manhã. Em relação às descompensações clínicas, de acordo com as informações colhidas, o paciente apresentou um episódio de crise adrenal no início do tratamento, fato que não ocorreu posteriormente, o que demonstra adequada adesão terapêutica, bem como supervisão e apoio parental. Além disso, considerando-se hábitos e costumes, constatouse prática de atividade física (vôlei e natação) e busca por uma alimentação mais saudável.

Durante o exame físico realizado na primeira consulta com atual endocrinologista pediátrico, foram verificados, conforme Tabela 1:

Tabela 1. Dados do exame físico, agosto de 2019.

\begin{tabular}{lc}
\hline Parâmetro & Resultado \\
\hline Altura & $163,5 \mathrm{~cm}(\mathrm{z}$ escore 0) \\
Índice de massa corpórea & $25,47 \mathrm{Kg} / \mathrm{m}^{2}(\mathrm{z}$ escore \\
(IMC) & $+1 /+2)$ \\
Pressão arterial & $100 \times 60 \mathrm{mmHg}$ (braço \\
& esquerdo) \\
Estadiamento de Tanner & $\mathrm{GPP} 3$ \\
Volume testicular & $6 \mathrm{~cm}^{3}$ \\
Comprimento peniano & $6 \mathrm{~cm}^{-}$ \\
Presença de acantose axilar & - \\
\hline
\end{tabular}

Fonte: Autores.
Em relação ao crescimento, segundo as informações apresentadas pela mãe do paciente, houve considerável incremento após a introdução do GH (hormônio do crescimento). Ademais, considerando-se a altura dos genitores (mãe $=178$ $\mathrm{cm}$ e pai $=171 \mathrm{~cm}$ ), a estatura-alvo para o paciente em questão foi calculada para $181 \mathrm{~cm}$, com uma variação de $176-186 \mathrm{~cm}$.

Para acompanhamento, foram solicitados novos exames de sangue e idade óssea. Em consultas subsequentes, observou-se ganho estatural, conforme exposto na Figura 3, e valores de IMC expostos na Figura 4.

\section{Aspectos éticos}

O paciente e seu responsável deram seu consentimento para participação na pesquisa. $O$ projeto foi submetido à avaliação e aprovado por um Comitê de Ética em Pesquisa.

\section{DISCUSSÃO}

O paciente descrito no presente relato apresentou inicialmente DI secundário à presença de germinoma suprasselar e, após a realização do tratamento para o mesmo, evoluiu com panhipopituitarismo. Em relação a isso, ressalta-se a importância do diagnóstico em tempo oportuno, com investigação apropriada e posterior seguimento direcionado para reposição adequada dos hormônios, manutenção da qualidade de vida, prevenção e manejo de complicações agudas ${ }^{8}$. 


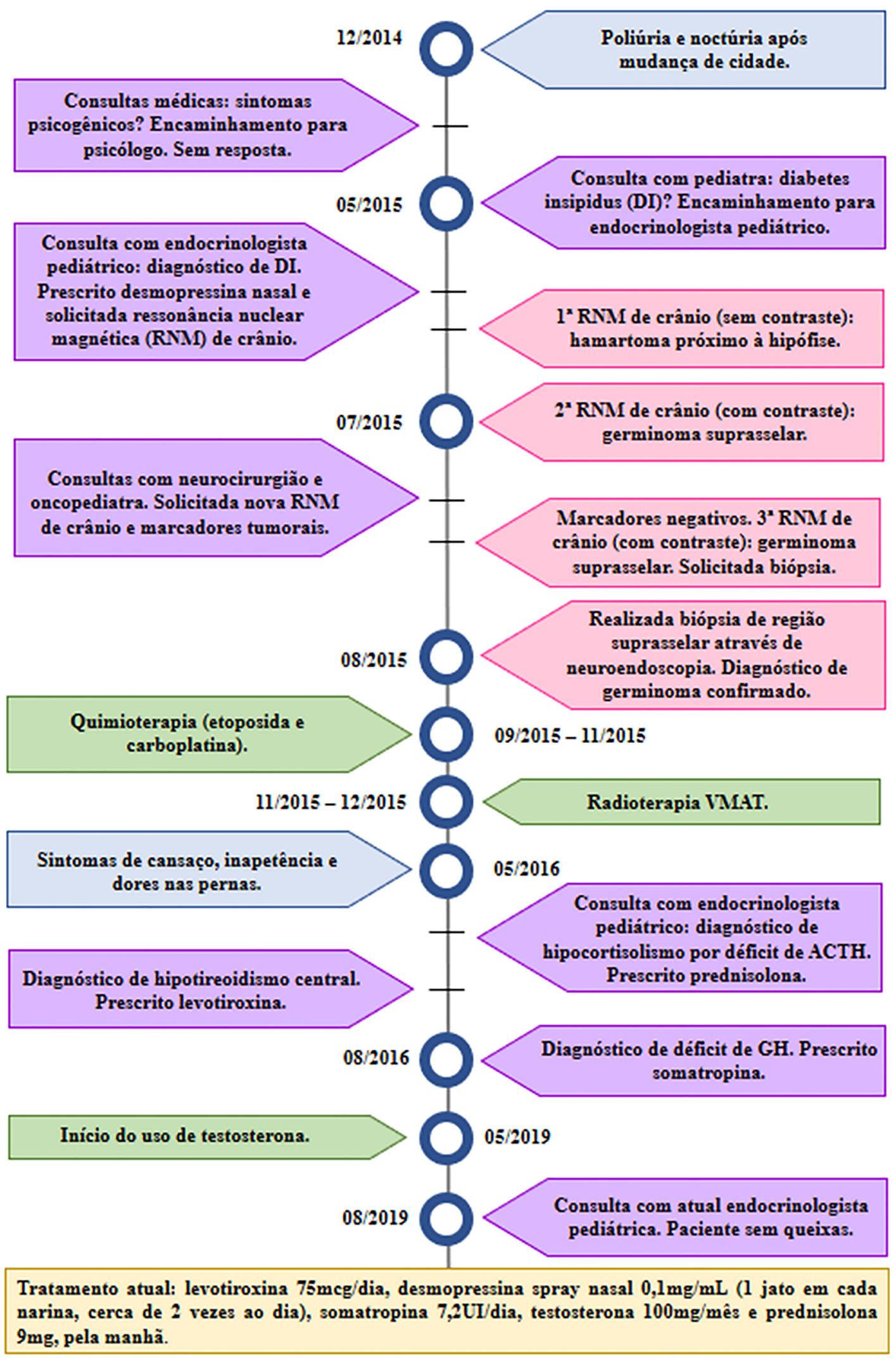

Figura 2. Linha do tempo da evolução do caso clínico. Fonte: Autores. 


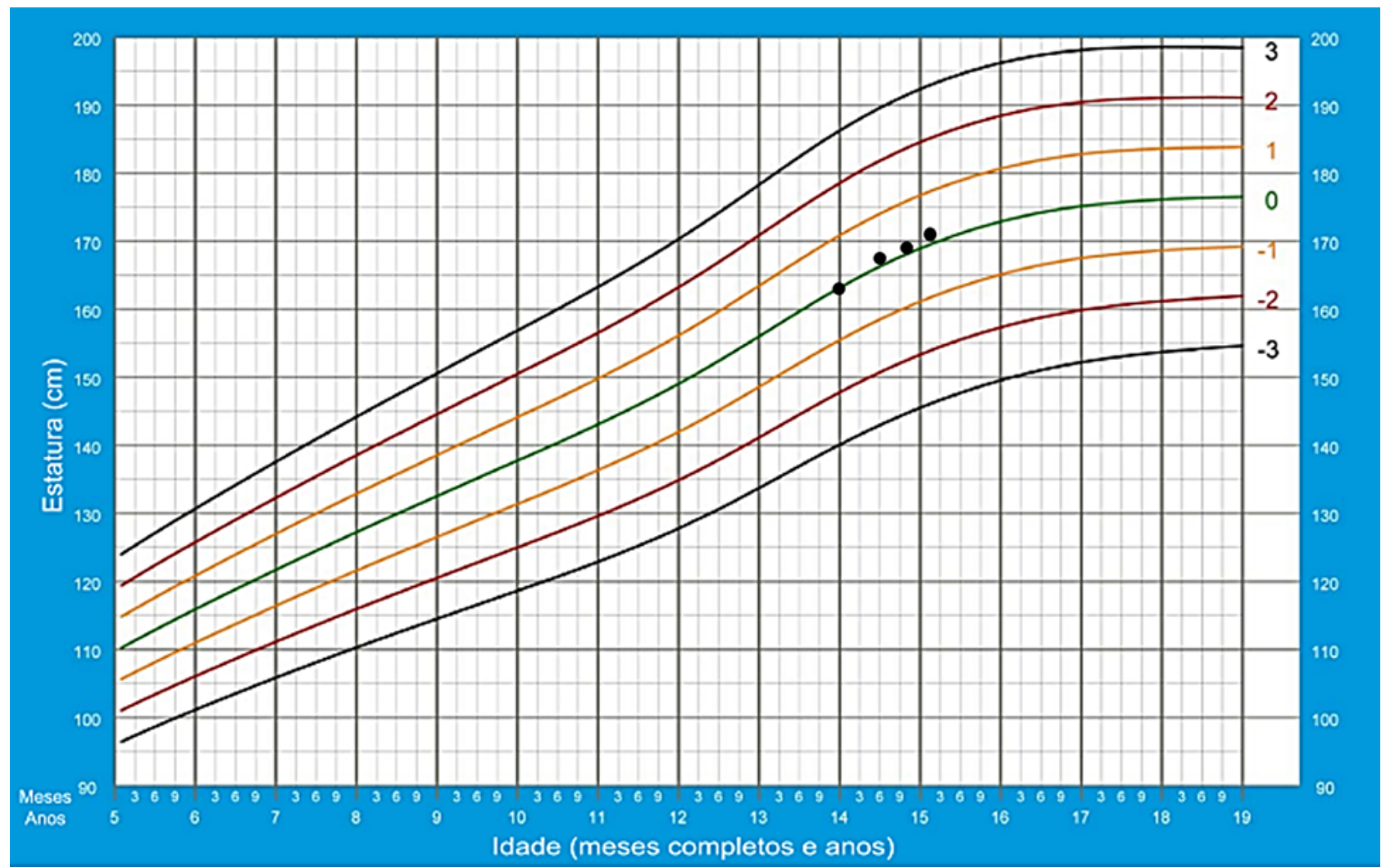

Figura 3. Estatura do paciente (em centímetros), de agosto de 2019 (aos 14 anos) a outubro de 2020 (aos 15 anos e 2 meses), conforme gráfico da OMS "Estatura por idade, meninos, dos 5 a 19 anos (escores-z). Fonte: Secretaria de Atenção Primária à Saúde, $2011^{9}$.

No tocante ao diagnóstico, os pacientes com DI apresentam-se clinicamente com queixas de poliúria e polidipsia; além disso, a presença de noctúria em indivíduo que dispunha de controle esfincteriano previamente também deve sugerir a possibilidade deste diagnóstico ${ }^{10}$. No paciente em questão, houve atraso no diagnóstico do DI, pensando tratar-se de quadro psicogênico, o que retardou o início do tratamento. Assim, diante da suspeita de poliúria, é importante a investigação com exames complementares.

Inicialmente, deve-se confirmar a poliúria, que é definida pelos valores diários de $120 \mathrm{ml} / \mathrm{kg}$ em recémnascidos, $100-110 \mathrm{ml} / \mathrm{kg}$ em lactentes e $40-50 \mathrm{ml} /$ $\mathrm{kg}$ em crianças maiores. Posteriormente, os exames laboratoriais necessários objetivam excluir distúrbios hidroeletrolíticos e estabelecer que se trata de poliúria hipotônica. Tais exames incluem osmolalidade sérica, sódio, potássio, glicose, cálcio, escórias nitrogenadas e avaliação urinária (osmolalidade, glicosúria e densidade específica $)^{10}$. Ademais, deve-se avaliar a presença prévia de hipotireoidismo e insuficiência adrenal, que podem ocultar o DI, devido à diminuição do clearance de água livre ${ }^{11}$.
A poliúria hipotônica, que caracteriza o DI, é expressa por baixa osmolalidade urinária (menor que $800 \mathrm{mOsm} / \mathrm{kg}$ ), bem como diminuída densidade urinária específica (menor que 1010). Em relação à polidipsia psicogênica, valores baixos de sódio plasmático (menor ou igual a $135 \mathrm{mmol} /$ litro) e osmolalidade sérica (menor ou igual a $280 \mathrm{mOsm} /$ $\mathrm{kg}$ ) são encontrados ${ }^{11}$.

Além disso, na situação de elevada osmolalidade plasmática e hipernatremia, a diferenciação entre causa central e nefrogênica do DI pode ser realizada mediante a administração subcutânea de análogo sintético da vasopressina, sendo que a consequente concentração urinária corresponde à etiologia central, conforme exposto no relato de caso. Em contraste, quando há valores normais de osmolalidade sérica e sódio em um contexto de poliúria hipotônica, é necessário teste de privação hídrica para elucidação da etiologia ${ }^{11}$.

Após a identificação de que o DI tem causa central, deve ser realizada RNM de crânio ${ }^{10}$, fato que também pode ser visualizado no manejo do caso clínico apresentado, em que foi demonstrado germinoma suprasselar. 


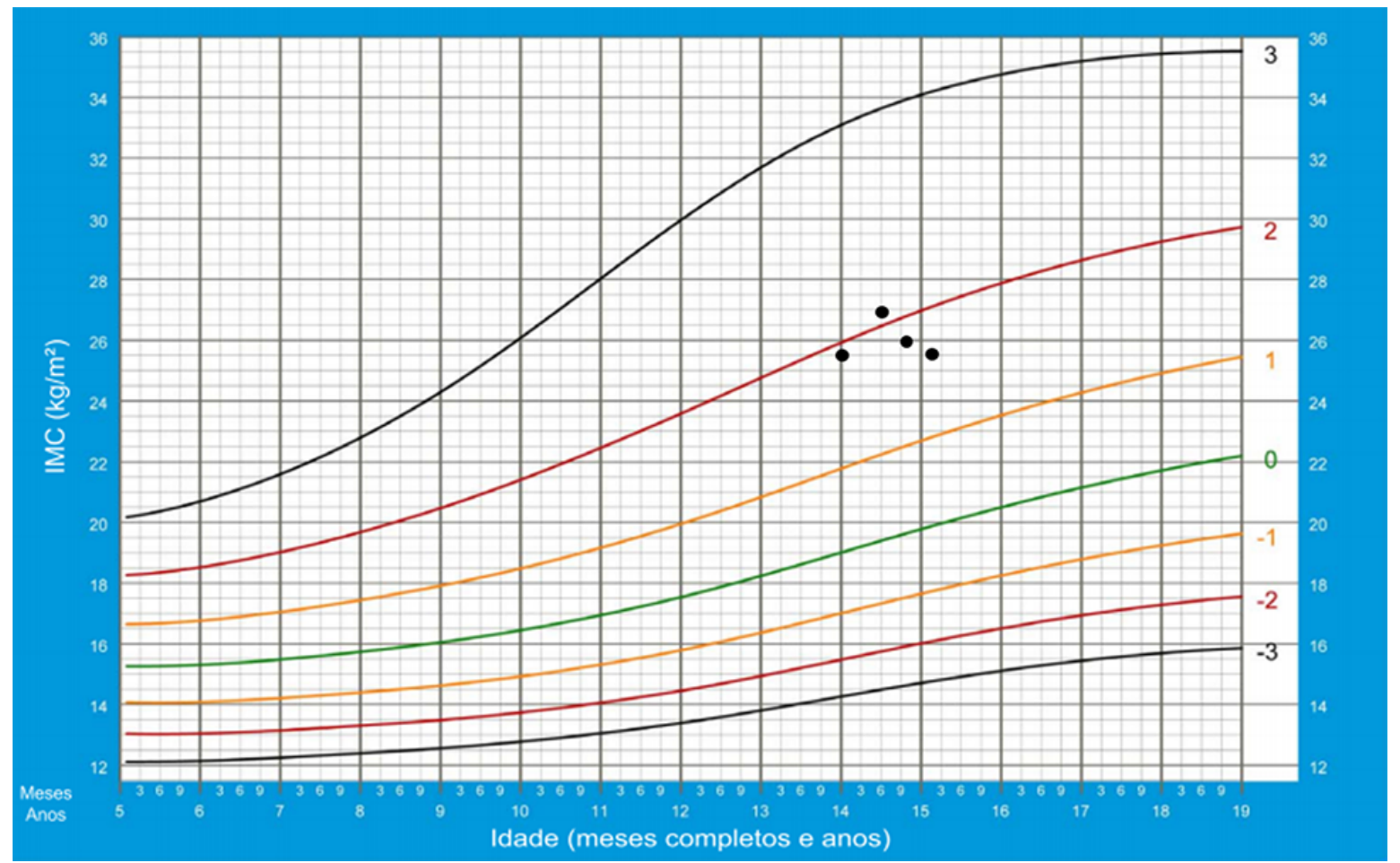

Figura 4. IMC do paciente (em quilograma por metro quadrado), de agosto de 2019 (aos 14 anos) a outubro de 2020 (aos 15 anos e 2 meses), conforme gráfico da OMS "IMC por idade, meninos, dos 5 a 19 anos (escores-z). Fonte: Secretaria de Atenção Primária à Saúde, $2011^{9}$.

Nesse cenário, considerando-se a normalidade dos eixos hipofisários previamente ao tratamento, notou-se o surgimento de deficiências hormonais posteriormente ao mesmo. Sob essa perspectiva, foi visto que doses de irradiação em área hipotalâmicahipofisária iguais ou superiores a $18 \mathrm{~Gy}$ já podem determinar déficit de $\mathrm{GH}$ e valores iguais ou maiores que 30Gy podem causar deficiência de TSH (hormônio estimulante da tireoide), ACTH, FSH (hormônio folículo-estimulante) e LH (hormônio luteinizante) ${ }^{12}$, o que ocorreu no paciente em questão.

Outro ponto que deve ser avaliado refere-se à manutenção da qualidade de vida. $\mathrm{O}$ adolescente do presente relato mantinha bom comportamento e aproveitamento escolar, bem como relacionamentos saudáveis, além de participar de atividades esportivas extracurriculares e desfrutar de opções alimentares saudáveis. Tal perspectiva deve ser continuamente verificada, visto que se percebe que adultos com deficiência múltipla de hormônios hipofisários de surgimento na infância apresentam pior qualidade de vida, com maior número de problemas de comportamento e desemprego, necessitando de acompanhamento em relação a questões biopsicossociais ${ }^{5}$.
Nesse cenário, é essencial a participação do endocrinologista no manejo dos déficits hormonais e avaliação periódica do tratamento ${ }^{6}$. Em relação ao tratamento hormonal na população pediátrica, sugerem-se as seguintes drogas e doses iniciais, conforme Tabela 2:

Tabela 2. Drogas de escolha e doses para as deficiências hormonais

\begin{tabular}{|c|c|c|}
\hline Deficiências & Droga & Doses \\
\hline $\mathrm{GH}$ & $\begin{array}{l}\text { Somatropina } \\
\text { subcutânea }{ }^{13}\end{array}$ & $\begin{array}{l}0,075-0,10 \mathrm{UI} / \\
\mathrm{kg} / \mathrm{dia}^{13}\end{array}$ \\
\hline TSH & $\begin{array}{l}\text { Levotiroxina oral } \\
(\mathrm{VO})^{14}\end{array}$ & $\begin{array}{l}100 \mu \mathrm{g} / \mathrm{m}^{2} / \mathrm{dia}, \\
15 \text { a } 30 \text { minutos } \\
\text { antes da primeira } \\
\text { refeição }{ }^{14}\end{array}$ \\
\hline $\mathrm{LH}$ e FSH & $\begin{array}{l}\text { Testosterona (para } \\
\text { o sexo masculino) } \\
\text { intramuscular }^{7}\end{array}$ & 50 mg/mês ${ }^{7}$ \\
\hline ACTH & Hidrocortisona oral ${ }^{7}$ & $7-9 \mathrm{mg} / \mathrm{m}^{2} / \mathrm{dia}^{7}$ \\
\hline $\begin{array}{l}\text { ADH } \\
\text { (hormônio } \\
\text { antidiurético) }\end{array}$ & Desmopressina $^{15}$ & $\begin{array}{l}\text { Oral: } 100 \text { a } 1200 \\
\mu g \text {, divididos em } \\
3 \text { vezes por dia. } \\
\text { Intranasal: } 2-40 \\
\mu g, 1 \text { ou } 2 \text { vezes } \\
\text { ao dia }{ }^{15}\end{array}$ \\
\hline
\end{tabular}


Ademais, considerando-se a descompensação decorrente do pan-hipopituitarismo e apresentada pelo paciente em questão no início do tratamento - crise adrenal -, ressalta-se a importância de que médicos dos prontos atendimentos a reconheçam. A crise adrenal é definida como agravo agudo à saúde, combinado à hipotensão ou taquicardia sinusal, ou a uma alteração eletrolítica ou hipoglicêmica significativa, que não é conferido a outra causa, e resolve-se após administração parenteral de glicocorticoides. Recomenda-se hidrocortisona intravenosa (IV) ou intramuscular (IM), em bolus, $4 \mathrm{mg} / \mathrm{kg}^{16}$.

Nesse cenário, a adolescência pode representar um desafio, visto que o paciente vai se tornando mais independente e assumindo, paulatinamente, a responsabilidade do tratamento. Assim, faz-se necessário incentivar sua autonomia, explicando-se sobre os fatores de risco para crises adrenais e o manejo das medicações ${ }^{7}$. Sugere-se também que o paciente tenha sempre consigo um cartão instrutivo, que alerte sobre sua doença e a possibilidade de crise adrenal ${ }^{16}$.

Finalmente, considerando-se o exposto sobre a relevância da reposição hormonal no manejo do pan-hipopituitarismo de surgimento na infância, percebe-se a importância da imediata suspeição clínica frente a manifestações sugestivas e da atenção que deve ser conferida durante a fase de adolescência. Observou-se que, no presente caso, houve atraso no diagnóstico e que, atualmente, o paciente apresenta-se assintomático e informado acerca do assunto, mostrando-se habilitado para o uso das medicações, além de possuir supervisão e apoio parental. Tais fatos, em conjunto com a avaliação periódica da terapêutica e monitorização do crescimento e desenvolvimento pelo endocrinologista pediátrico, estão sendo fundamentais para a manutenção da qualidade de vida do adolescente.

\section{REFERÊNCIAS}

1. Ribeiro-Oliveira Júnior, A; Naves, LA; Vilar, L. Hipopituitarismo: etiologia e diagnóstico. In: Vilar, L, editor. Endocrinologia Clínica. Rio de Janeiro: Guanabara Koogan; 2017. p. 35-48.

2. Yeliosof, O; Gangat, M. Diagnosis and management of hypopituitarism. Current Opinion in Pediatrics. 2019; 31(4):531-6. Disponível em: https://journals.Iww.com/ co-pediatrics/Abstract/2019/08000/Diagnosis_and_ management_of_hypopituitarism.17.aspx.

3. Pekic, S; Popovic, V. Diagnosis of endocrine disease: expanding the cause of hypopituitarism. European Journal of Endocrinology. 2017; 176(6):269-282. Disponível em: https://pubmed.ncbi.nlm.nih.gov/28258131/.

4. Webb, EA; Dattani, MT. Understanding hypopituitarism. Paediatrics and Child Health. 2015; 25(7):295-301. Disponível em: https://www. paediatricsandchildhealthjournal.co.uk/article/S17517222(15)00069-4/pdf.

5. Kao, KT; Stargatt, R; Zacharin, M. Adult Quality of Life and Psychosocial Outcomes of Childhood Onset Hypopituitarism. Hormone Research In Paediatrics. 2015; 84(2):94-101. Disponível em: https://www.ncbi. nlm.nih.gov/pubmed/26045297.

6. Higham, CE; Johannsson, G; Shalet, SM. Hypopituitarism. The Lancet. 2016; 388(10058):2403-15. Disponível em: https://www.ncbi.nlm.nih.gov/pubmed/27041067.

7. Sbardella, E; Pozza C; Isidori, AM; Grossman, AB. Endocrinology and adolescence: dealing with transition in young patients with pituitary disorders. European Journal of Endocrinology. 2019; 181(4):R155-R171. Disponível em: https://eje.bioscientifica.com/abstract/ journals/eje/181/4/EJE-19-0298.xml.

8. Alexandraki, K; Grossman, AB. Management of hypopituitarism. Journal of Clinical Medicine. 2019; 8(12):1-23. Disponível em: https://pubmed.ncbi.nlm. nih.gov/31817511/.

9. Secretaria de Atenção Primária à Saúde (SAPS). Curvas de Crescimento da Organização Mundial da Saúde - OMS [Internet]. Brasília: Ministério da Saúde [atualizado em 2011; acesso em 08 mar 2021]. Disponível em: https://aps.saude.gov.br/ape/vigilanciaalimentar/ curvascrescimento.

10. Dabrowski, E; Kadakia, R; Zimmerman, D. Diabetes insipidus in infants and children. 2016; 30(2):317328. Disponível em: https://pubmed.ncbi.nlm.nih. gov/27156767/.

11. Elias, PCL; Castro, M; Moreira, AC. Diabetes Insípido: visão geral. In: Vilar, $L$, editor. Endocrinologia Clínica. Rio de Janeiro: Guanabara Koogan; 2017. p. 172-185.

12. Chemaitilly, W; Cohen, LE; Mostoufi-Moab, S; Patterson, BC; Simmons, JH; Meacham, LR et al. Endocrine Late Effects in Childhood Cancer Survivors. Journal of Clinical Oncology. 2018; 36(21):2153-2159. Disponível em: https://pubmed.ncbi.nlm.nih.gov/29874130/.

13. Grimberg, A; DiVall, SA; Polychronakos, C; Allen, DB; Cohen, LE; Quintos, JB et al. Guidelines for Growth Hormone and Insulin-Like Growth Factor-I Treatment in Children and Adolescents: Growth Hormone Deficiency, Idiopathic Short Stature, and Primary Insulin-Like Growth Factor-I Deficiency. Hormone Research In Paediatrics. 2016; 86(6):361-97. Disponível em: https://www.ncbi.nlm.nih.gov/pubmed/27884013.

14. Jonklaas, J; Bianco, AC; Bauer, AJ; Burman, KD; Cappola, AR; Celi, FS et al. Guidelines for the Treatment of Hypothyroidism. Thyroid. 2014; 24(12):1670-1751. Disponível em: https://www.ncbi.nlm.nih.gov/pmc/ articles/PMC4267409/. 
15. Di Iorgi, N; Napoli, F; Allegri, AEM; Olivieri, I; Bertelli, E; Gallizia, A et al. Diabetes Insipidus: diagnosis and management. Hormone Research In Paediatrics. 2012. 77(2):69-84. Disponível em: https://www.ncbi.nlm.nih. gov/pubmed/22433947.
16. Rushworth, RL; Torpy, DJ; Stratakis, CA; Falhammar, $\mathrm{H}$ et al. Adrenal crisis in children: perspectives and research directions. Hormone Research in Paediatrics. 2018; 89(5):341-351. Disponível em: https://pubmed. ncbi.nlm.nih.gov/29874655/. 


\section{Contribuições específicas de cada autor}

- $\quad$ Na concepção OU desenho do trabalho: Vivianne A.N.; R.C.V.L.S.;

- $\quad$ Na redação OU revisão crítica com contribuição intelectual: Vivianne A.N.; Vinícius A.N.; R.C.V.L.S.;

- $\quad$ Na aprovação final da versão para publicação: Vivianne A.N.; Vinícius A.N.; R.C.V.L.S.

Fontes de apoio ou financiamento

não houve fontes de apoio ou financiamento que tenham contribuído para o desenvolvimento do trabalho.

Autor Correspondente:

Vivianne Almeida da Nóbrega

vivianne8nobrega@gmail.com

Editor:

Prof. Paulo Henrique Manso

Recebido: 28/11/2020

Aprovado: 21/05/2021 